\title{
Lack of association between the cyclooxygenase 2 -765G >C polymorphism and prostate cancer risk: a meta-analysis
}

\author{
Y.-Q. Feng ${ }^{1}$, Y.U. Li' ${ }^{2}$, W.-D. Xiao', G.-X. Wang ${ }^{2}$ and Y.O. Li ${ }^{1}$ \\ 1'Department of General Surgery, The First Affiliated Hospital of Nanchang University, \\ Nanchang, Jiangxi Province, China \\ ${ }^{2}$ Department of Urologic Surgery, Jiangxi Institute of Urology, \\ The First Affiliated Hospital of Nanchang University, Nanchang, Jiangxi Province, \\ China \\ Corresponding author: Y.O. Li \\ E-mail: yfyly@163.com \\ Genet. Mol. Res. 14 (4): 13391-13402 (2015) \\ Received February 23, 2015 \\ Accepted July 14, 2015 \\ Published October 28, 2015 \\ DOI http://dx.doi.org/10.4238/2015.October.28.1
}

\begin{abstract}
The aim of this study was to investigate the association between the cyclooxygenase 2 (COX2) $\quad-765 \mathrm{G}>\mathrm{C}$ (rs20417) polymorphism and prostate cancer (PC) risk using meta-analysis. A systematic literature search was performed using the PubMed, Embase, Cochrane Library, and Google Scholar databases by using the terms "cyclooxygenase-2/COX-2/PTGs2", "polymorphism" or "variation", and "prostate" and "cancer" or "carcinoma" to identify relevant articles up to June 14, 2014. Crude odds ratios (ORs) with 95\% confidence intervals (Cls) were assessed for PC risk associated with COX2 -765G>C polymorphism using fixed- and random-effect models. We identified a total of nine publications, including 5952 cases and 5078 controls, to investigate the effect of $C O X 2-765 \mathrm{G}>\mathrm{C}$ on $\mathrm{PC}$ risk, and found no significant association in any genetic model tested (CC vs GG: OR = $0.993,95 \% \mathrm{Cl}=0.923-1.068 ; \mathrm{GC}+\mathrm{CC}$ vs GG: $\mathrm{OR}=1.041,95 \% \mathrm{Cl}=$ 0.931-1.103; CC vs GC+GG: $\mathrm{OR}=0.858,95 \% \mathrm{Cl}=0.689-1.067 ; \mathrm{CC}$
\end{abstract}


vs $\mathrm{GG}: \mathrm{OR}=0.871,95 \% \mathrm{Cl}=0.689-1.086 ; \mathrm{GC}$ vs $\mathrm{GG}: \mathrm{OR}=1.032$, $95 \% \mathrm{Cl}=0.945-1.127)$. Power analysis and tests for publication bias ensured the reliability of our results. This meta-analysis suggested that the functional COX2 -765G>C polymorphism, located in the COX2 gene promoter, is unlikely to be associated with $\mathrm{PC}$ risk. However, additional larger, well-designed studies are still required to reach a conclusive result on this issue.

Key words: COX2 -765G>C; Polymorphism; Prostate cancer; Meta-analysis

\section{INTRODUCTION}

Prostate cancer $(\mathrm{PC})$ is one of the most prevalent malignancies in men and the second most frequent cause of male cancer-related death (Siegel et al., 2012). It is a clinically heterogeneous disease and the incidence is increasing. PC is also a major public health burden in many countries, and seriously impacts patient quality of life.

Cyclooxygenase (COX), also known as prostaglandin-endoperoxide synthase (PTGS), is a rate-limiting enzyme produced during the production of prostaglandins (Wang et al., 2007). There are two forms of COX proteins, COX-1 and COX-2; COX-1 may have a role as a housekeeping enzyme involved in cell signaling, whereas COX-2 is absent from many cell types unless induced by tumor promoters, growth factors, or cytokines (Kawata et al., 1995). More importantly, COX2 is also involved in mechanisms of carcinogenesis such as apoptosis (Ding et al., 2000; Li et al., 2001), invasiveness (Sheng et al., 2001), adhesion (Chen et al., 2001), and angiogenesis (Masferrer et al., 2000).

The single nucleotide polymorphism (SNP) COX2 -765G>C (rs20417) is a functional, extensively studied polymorphism that consists of a guanine $(G)$ to cytosine $(C)$ conversion at position -765 of the COX2 promoter region, altering the transcriptional activity of the COX2 gene. Over the last decade, numerous molecular epidemiological case-control studies have been conducted in diverse ethnic backgrounds to explore the association between the COX2 -765G>C polymorphism and risks of various cancers, including breast cancer (Piranda et al., 2010), colorectal carcinoma (Daraei et al., 2012), esophageal (Bye et al., 2011) and gastric cancers (Li et al., 2012), hepatocellular carcinoma (He et al., 2012), leukemia (Zheng et al., 2011), lung cancer (Coskunpinar et al., 2011), lymphoma (Monroy et al., 2011), ovarian (Agachan Cakmakoglu et al., 2011), head and neck (Peters et al., 2009), pancreatic (Zhao et al., 2009), skin (Cocoş et al., 2012), and cervical cancers (Pandey et al., 2010), among others. However, due to the relatively small sample sizes, the results obtained from these studies have been inconclusive or even controversial. In 2010, a meta-analysis was conducted with the aim to assess the exact relationship of the COX2 -765G>C polymorphism with PC susceptibility (Murad et al., 2009), and demonstrated that it was associated with increased risk of PC. However, there were some limitations of this meta-analysis, such as a relatively small number of studies included (only two in addition to the author's own study) and incorrectly extracted data.

In consideration of the small sample sizes utilized in previous studies, we performed this meta-analysis to systematically summarize the published data to assess the association between COX2 -765G $>C$ and PC. 


\section{MATERIAL AND METHODS}

\section{Publication search}

A systematic literature search was performed using the PubMed, Embase, Cochrane Library, and Google Scholar databases by using the terms "cyclooxygenase-2/COX-2/PTGs2", "polymorphism" or "variation", and "prostate" and "cancer" or "carcinoma" to identify relevant articles up to June 14,2014 . We evaluated all associated publications to retrieve the most eligible literature. In addition, the reference lists in each database were hand-searched to find other relevant publications. Articles were limited to English language papers.

\section{Inclusion and exclusion criteria}

Studies were selected if they met the following criteria: 1) published in peer-reviewed journals, 2) about the COX2 $-765 \mathrm{G}>\mathrm{C}$ polymorphism and risk of $\mathrm{PC}$, and 3) contained useful genotype frequencies. The exclusion criteria were: 1) non-case-control studies, 2) control population included malignant tumor patients, 3 ) the genotype frequencies of the control group departed from Hardy-Weinberg equilibrium (HWE), and 4) duplicated publications.

\section{Data extraction}

Two investigators (Y.Q.F. and Y.L.) reviewed and extracted information from all eligible publications independently, according to the inclusion and exclusion criteria listed above. An agreement was reached by discussion between the two reviewers whenever there was a conflict. The following items were collected from each study: first author's surname, year of publication, country of origin, ethnicity of participants, source of controls used for assessment of COX2-765G>C genotypes, and total number of cases and controls as well as numbers of cases and controls with $\mathrm{C} / \mathrm{C}, \mathrm{G} / \mathrm{C}$, and $\mathrm{G} / \mathrm{G}$ genotypes.

\section{Statistical analysis}

Crude odds ratios (ORs) with $95 \%$ confidence intervals (Cls) were used to assess the association between the COX2 $-765 \mathrm{G}>\mathrm{C}$ polymorphism and PC risk. The pooled ORs were performed for homozygote comparison (CC vs GG), and dominant (CC + GC vs GG), recessive (CC vs $C G+G G$ ), and codominant models (CC vs GG, CG vs GG). Stratified analyses were performed based on the source of controls, the specimens used for determining COX2 -765G>C genotypes, and racial descent. A chi-square-based Q-test was performed to check the heterogeneity (Cochran, 1954). If $P \geq 0.1$ was obtained in the heterogeneity test, ORs were pooled according to the fixed-effect model (the Mantel-Haenszel model) (Mantel and Haenszel, 1959), otherwise the random-effect model (the DerSimonian and Laird model) was used (DerSimonian and Laird, 1986). One-way sensitivity analyses were performed to evaluate the stability of the meta-analysis results. Potential publication bias was estimated using Begg's funnel plot and the Egger test; $P<0.05$ was considered to be statistically significant for publication bias (Begg and Mazumdar, 1994; Egger et al., 1997). If publication bias existed, the Duval and Tweedie non-parametric "trim-and-fill" method was used to adjust the results accordingly (Duval and Tweedie, 2000). All statistical tests were performed with the STATA software, version 12.1 (Stata Corporation; College Station, TX, USA). 


\section{RESULTS}

\section{Study characteristics}

The initial search yielded 78 studies; 61 were excluded after review of the title or abstract. After screening the full text of the remaining 16 studies, and from the extraction of data, five articles that were not case-control studies, and one review article were excluded. One study (Dossus et al., 2010) was excluded because the genotype frequencies of controls deviated from HWE. Thus, a total of 9 case-control studies [Panguluri et al., 2004; Cheng et al., 2007; Murad et al., 2009; Balistreri et al., 2010; Wu et al., 2011; Catsburg et al., 2012; Joshi et al., 2012; with Panguluri et al. (2004) and Cheng et al. (2007) including two studies], involving 5078 cases and 5952 controls, were included in the present meta-analysis. Figure 1 provides a summary of the selection process. Patient data were compiled from six Caucasian studies (Panguluri et al., 2004; Cheng et al., 2007; Murad et al., 2009; Balistreri et al., 2010; Catsburg et al., 2012; Joshi et al., 2012), 2 African studies (Panguluri et al., 2004; Cheng et al., 2007), and 1 Asian study (Wu et al., 2011).

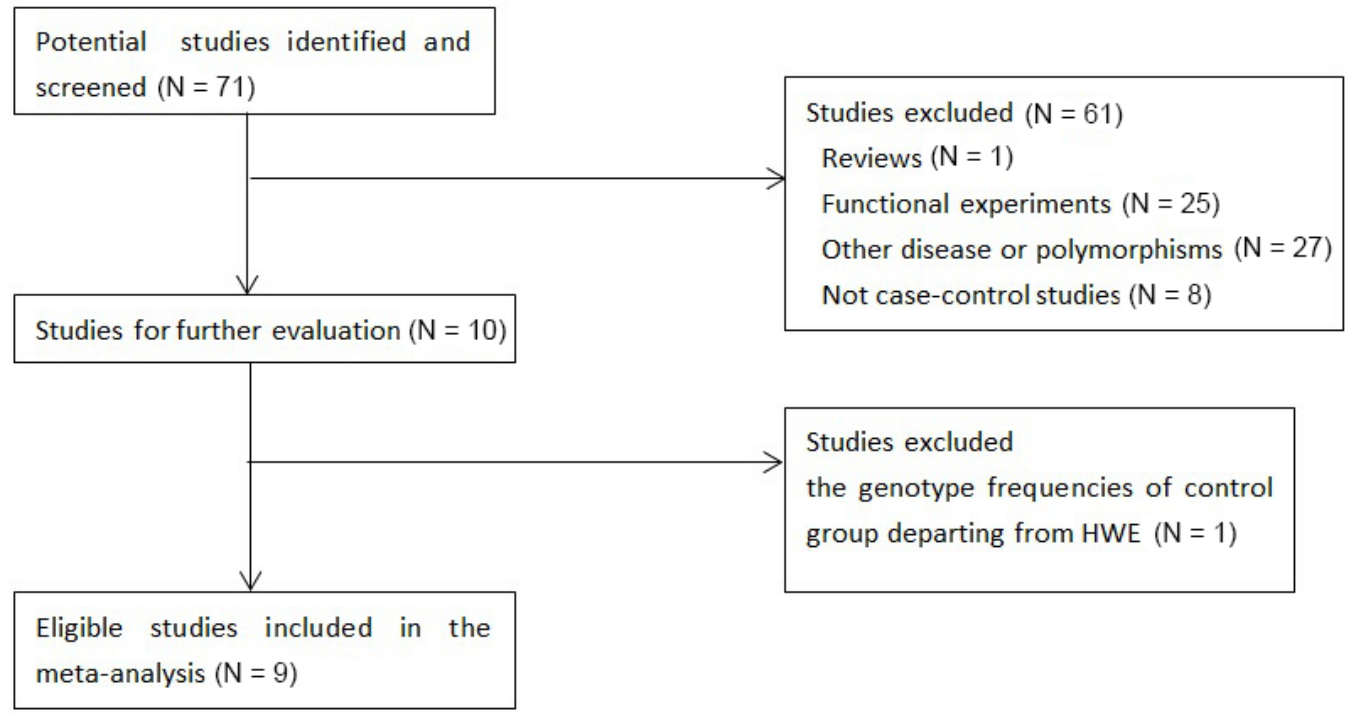

Figure 1. Study identification, inclusion, and exclusion for meta-analysis.

The characteristics of the nine eligible case-control studies are summarized in Table 1. The sample size of the nine studies ranged from 177 to 4620 in total, and a total of 5078 patients with PC and 5952 controls were included in the meta-analysis. The distribution of COX2 $-765 \mathrm{G}>\mathrm{C}$ genotype frequencies among patients with $\mathrm{PC}$ and controls from the 9 studies are shown in Table 2. Five studies's control group are from hospital which were patients who did not have PC, another 4 studies' control group are from normal people which were chosen randomly. 
Table 1. Main characteristics of studies included in the meta-analysis.

\begin{tabular}{|c|c|c|c|c|c|}
\hline First author (year) & Country & Ethnicity & Source of controls & Sample size (case/control) & HWE \\
\hline Balistreri et al. (2010) & Italy & Caucasian & $\mathrm{HB}$ & $50 / 125$ & $\mathrm{Y}$ \\
\hline Cheng et al. (2007) & US & Caucasian & $\mathrm{HB}$ & $416 / 417$ & Y \\
\hline Cheng et al. (2007) & US & African & $\mathrm{HB}$ & $89 / 88$ & Y \\
\hline Murad et al. (2009) & UK & Caucasian & $\mathrm{HB}$ & $1592 / 3028$ & Y \\
\hline Catsburg et al. (2012) & US & Caucasian & PB & $1431 / 756$ & Y \\
\hline Wu et al. (2011) & China & Asian & $\mathrm{HB}$ & $218 / 436$ & Y \\
\hline Joshi et al. (2012) & US & Caucasian & PB & $935 / 756$ & Y \\
\hline Panguluri et al. (2004) & Nigeria & Caucasian & PB & $87 / 90$ & Y \\
\hline Panguluri et al. (2004) & Nigeria & African & PB & $260 / 256$ & Y \\
\hline
\end{tabular}

HWE = Hardy-Weinberg equilibrium; US = United States; UK = United Kingdom; HB = hospital-based; PB = populationbased.

Table 2. Distribution of COX2 $-765 \mathrm{G}>\mathrm{C}$ genotypes among patients with prostate cancer and controls.

\begin{tabular}{|c|c|c|c|c|c|c|}
\hline \multirow[t]{2}{*}{ First author (year) } & \multicolumn{3}{|c|}{ Patients $(\mathrm{N})$} & \multicolumn{3}{|c|}{ Controls $(\mathrm{N})$} \\
\hline & GG & GC & $\mathrm{CC}$ & GG & GC & $\mathrm{CC}$ \\
\hline Balistreri et al. (2010) & 31 & 15 & 4 & 65 & 46 & 14 \\
\hline Cheng et al. (2007) & 294 & 115 & 7 & 293 & 113 & 11 \\
\hline Cheng et al. (2007) & 38 & 42 & 9 & 38 & 38 & 12 \\
\hline Murad et al. (2009) & 1104 & 451 & 37 & 2137 & 819 & 72 \\
\hline Catsburg et al. (2012) & 892 & 469 & 70 & 481 & 237 & 38 \\
\hline Wu et al. (2011) & 198 & 20 & 0 & 365 & 71 & 0 \\
\hline Joshi et al. (2012) & 595 & 304 & 36 & 481 & 237 & 38 \\
\hline Panguluri et al. (2004) & 86 & 1 & 0 & 88 & 2 & 0 \\
\hline Panguluri et al. (2004) & 202 & 52 & 6 & 205 & 42 & 9 \\
\hline
\end{tabular}

\section{Meta-analysis results}

All nine studies included, with a total of 5952 patients and 5078 controls, were pooled to explore the association between COX2 $-765 \mathrm{G}>\mathrm{C}$ and the susceptibility to PC. Detailed results are summarized in Table 3. Overall, there was no significant heterogeneity in any of the genetic models, and a fixed-effect model was applied to estimate the ORs. We did not find a statistical association of COX2 $-765 \mathrm{G}>\mathrm{C}$ with PC risk in the allelic model (CC vs GG: OR $=0.993,95 \% \mathrm{Cl}=$ 0.923-1.068) (Figure 2A). Similar results were observed in the other models (GC+CC vs GG: OR $=1.041,95 \% \mathrm{Cl}=0.931-1.103 ; \mathrm{CC}$ vs $\mathrm{GC}+\mathrm{GG}: \mathrm{OR}=0.858,95 \% \mathrm{Cl}=0.689-1.067 ; \mathrm{CC}$ vs GG: $\mathrm{OR}=0.871,95 \% \mathrm{Cl}=0.689-1.086 ; \mathrm{GC}$ vs GG: $\mathrm{OR}=1.032,95 \% \mathrm{Cl}=0.945-1.127$ ) (Figure 2B$E)$. In subgroup analysis by ethnicity and source of control group, nearly all of the genetic models demonstrated non-significant results. Although the homozygote comparison (CC vs GG) and the dominant model $(G C+C C$ vs $G G$ ) analyses from the Asian studies appeared to show that the $C$ allele conferred a higher $P C$ risk than did the $G G$ genotype $(O R=0.519,95 \% \mathrm{Cl}=0.307-0.878)$, this result was based on a small sample size (218 patients and 436 controls), and discrepancy with the other models made it difficult to have confidence in the reliability of this finding.

\section{Evaluation of study quality}

A single study involved in the meta-analysis was deleted in successive iterations to reflect the effects of individual data sets on the pooled ORs; most of the corresponding pooled ORs were not materially altered (data not shown). 


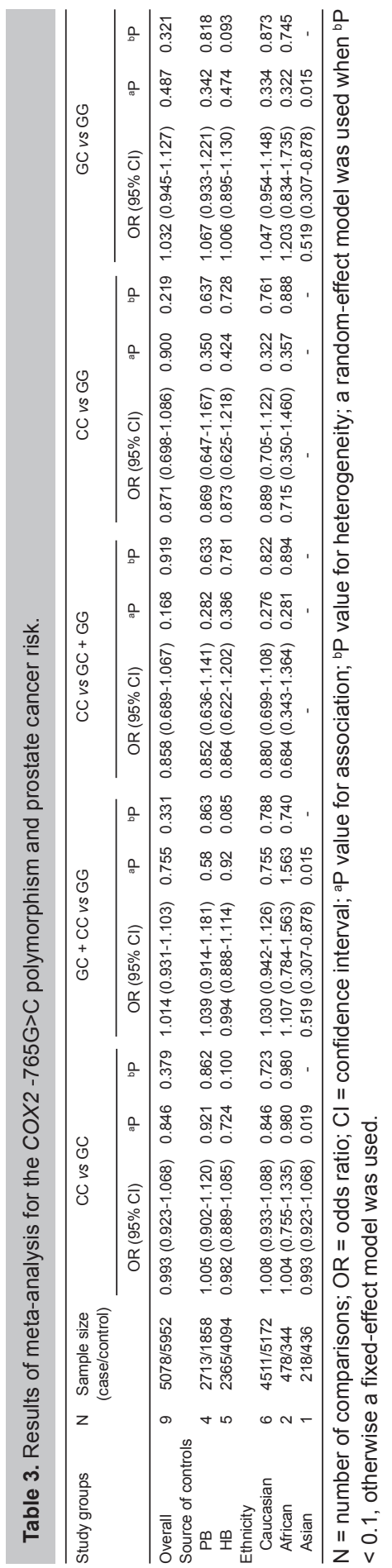


A

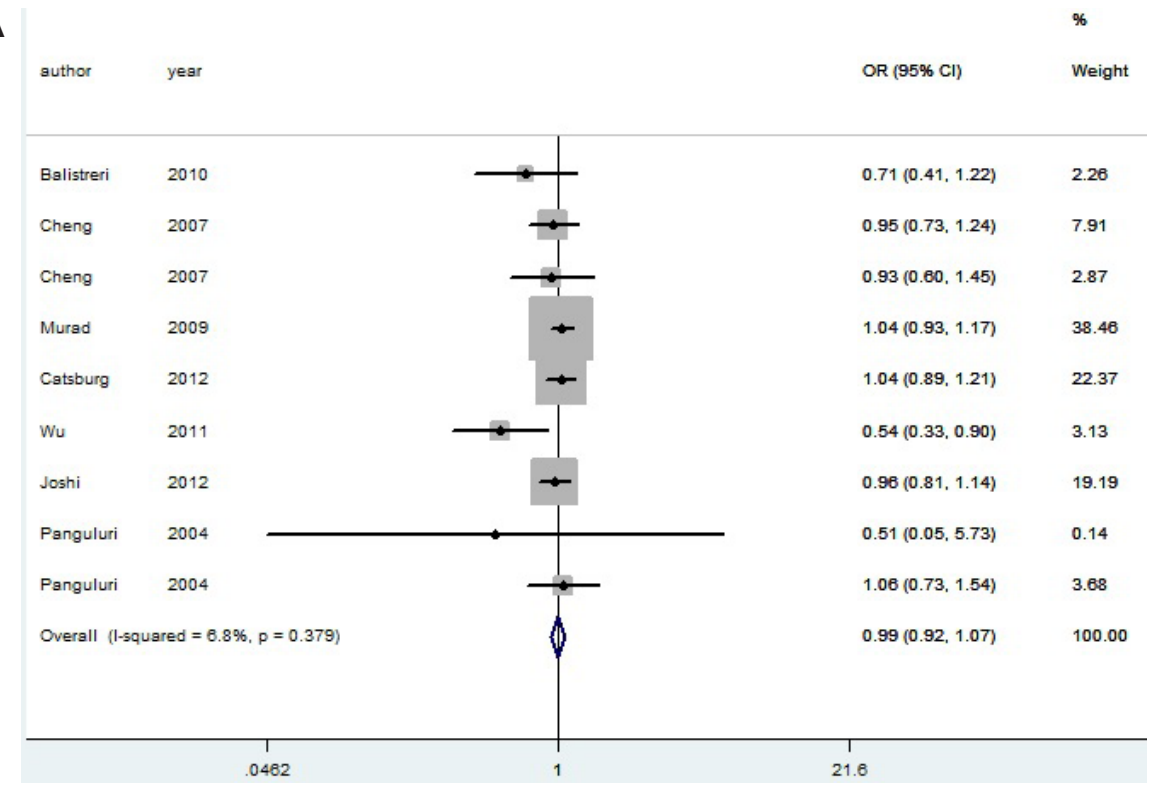

B

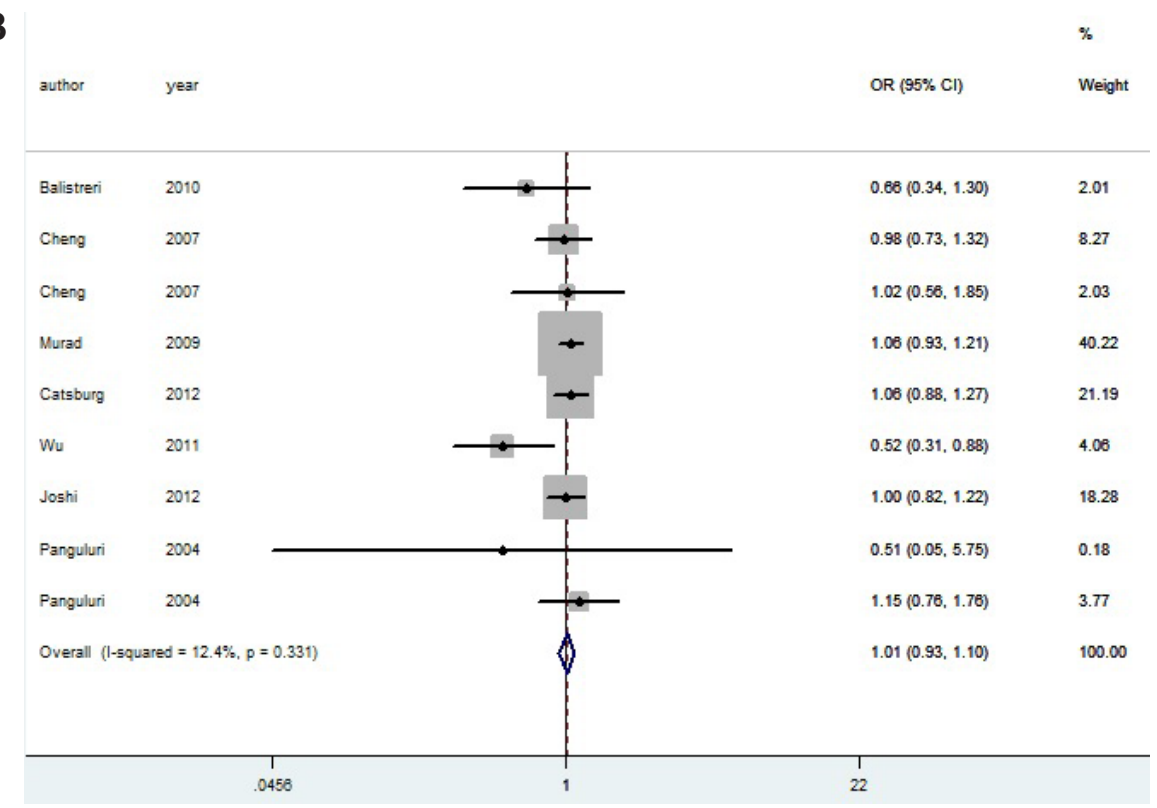

Figure 2. Forest plots of the relationships between the COX2-765G $>C$ polymorphism and prostate cancer risk in studies included. A. Homozygote comparison (CC vs GG). B. Dominant model (CC + GC vs GG). C. Recessive model (CC vs CG + GG). D. E. Codominant model (CC vs GG, CG vs GG, respectively). The first author surname and year of publication are given in the left side of each figure. The size of the grey square corresponding to each study is proportional to the sample size. The center of each square represents the odds ratio (OR) and the horizontal line shows the corresponding $95 \%$ confidence interval $(\mathrm{Cl})$. The pooled OR was obtained using a fixed-effect model and is represented by an open diamond, with the center indicating the OR and the ends corresponding to the $95 \% \mathrm{Cl}$.

Continued on next page 
Figure 2. Continued.

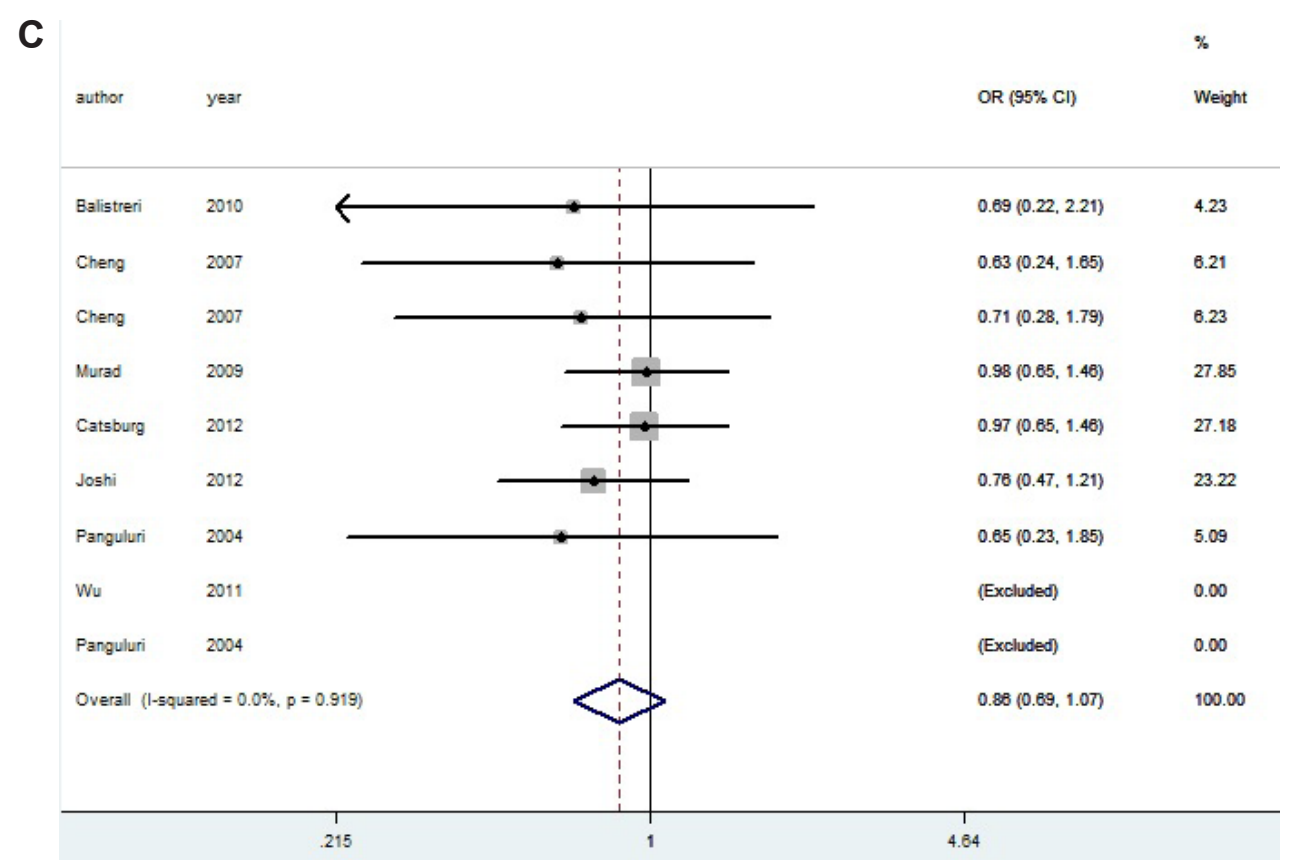

D

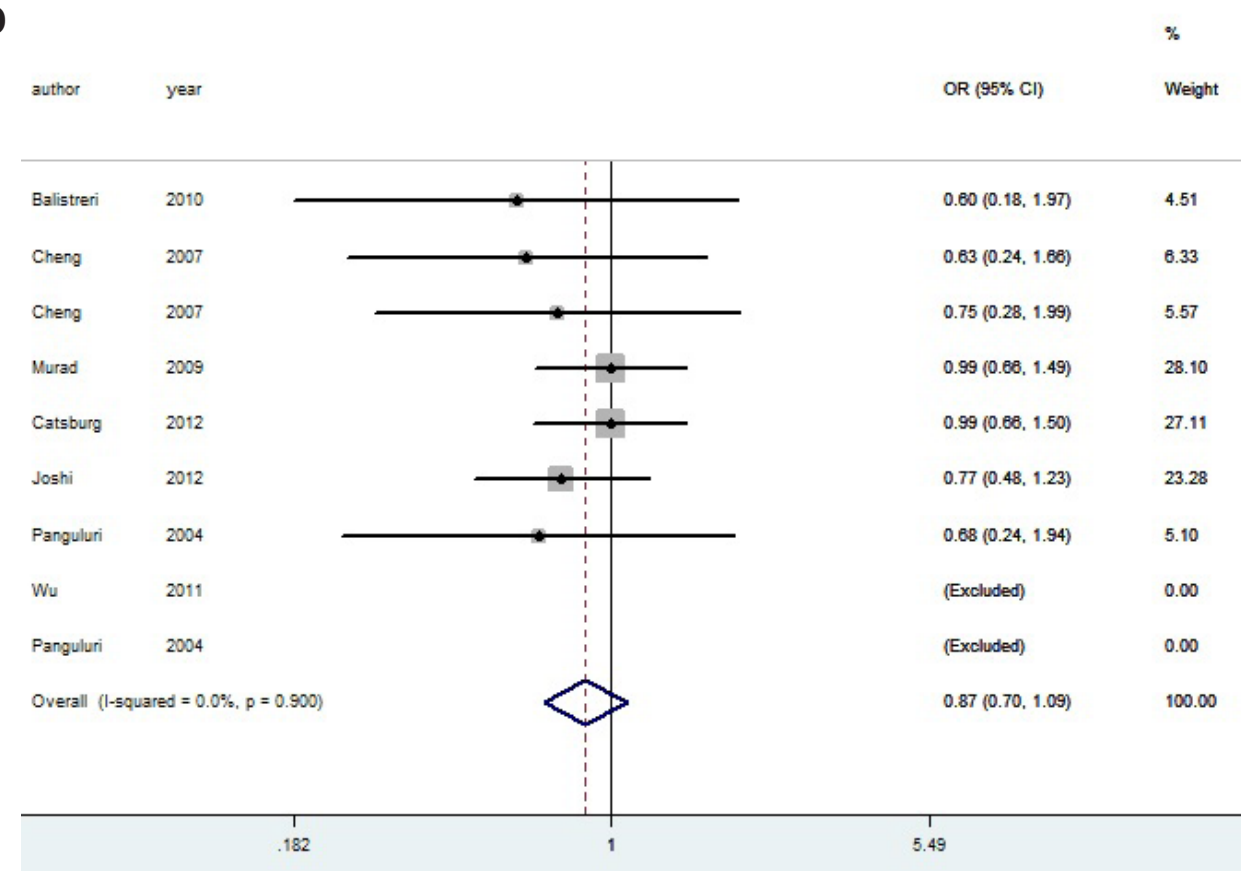

Continued on next page 
Figure 2. Continued.

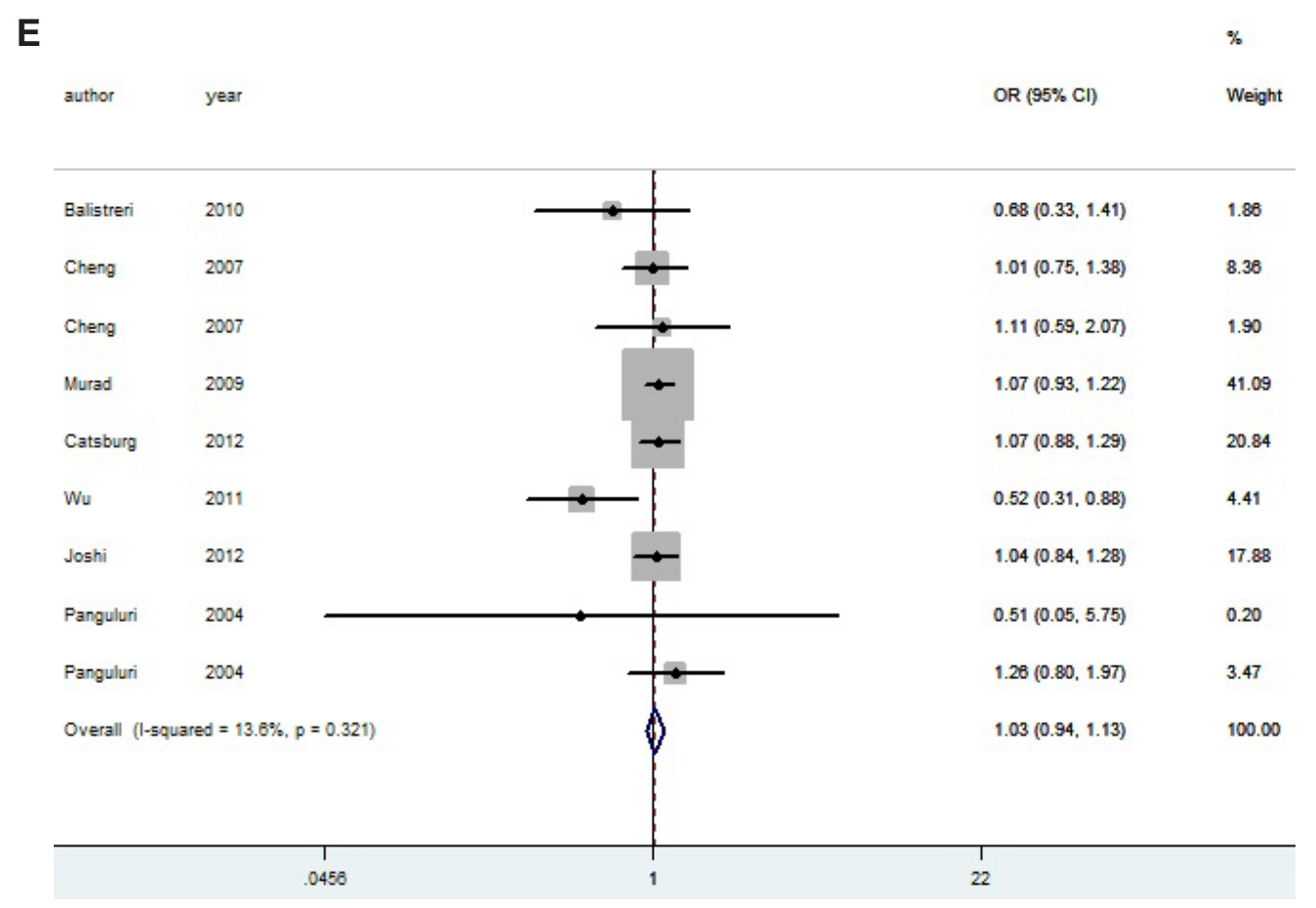

Both Begg's funnel plot and the Egger test were conducted to assess the publication bias of the currently available literature (Table 4). The Egger test results suggested that publication bias was evident in the heterozygote comparison $(P=0.038)$. Therefore, the Duval and Tweedie non-parametric "trim-and-fill" method was used to adjust for publication bias. Meta-analyses with and without using the "trim-and-fill" method did not draw different conclusions (data not shown). The shapes of the funnel plots were roughly symmetrical in the other comparison models (data not shown). Together, these findings indicated that our results were statistically robust.

Table 4. Statistical analysis of publication bias for $\mathrm{COX} 2-765 \mathrm{G}>\mathrm{C}$ polymorphism association with prostate cancer risk.

\begin{tabular}{lccccc}
\hline Category & CC vs GG & GC + CC vs GG & CC vs GC + GG & CC vs GG & GC vs GG \\
\hline Begg's test & 0.404 & 0.211 & 0.881 & 0.300 & 0.458 \\
Egger's test & 0.038 & 0.121 & 0.347 & 0.111 & 0.379 \\
\hline
\end{tabular}

\section{DISCUSSION}

COX2 $-765 G>C$ is a functional polymorphism, located at $765 \mathrm{bp}$ upstream (-765 bp) from the transcription start site of the COX2 gene. The polymorphic variant changes a putative stimulatory protein-binding site in the promoter of COX2 between -766 and -761 bp (Papafili et al., 2002), and it also creates an E2 promoter factor-binding site, leading to high COX2 transcription 
activity, which may be the mechanism of the COX2 -765G>C polymorphism increasing cancer risk (Szczeklik et al., 2004).

To explore the exact association between the COX2 -765G>C polymorphism and cancer risk, a meta-analysis (Murad et al., 2009) had been conducted in 2009; however, only the author's own work and two additional studies were contained in that meta-analysis, and due to the relatively small overall sample size and deficits in data collection, the results were conflicting. Therefore, we conducted the current meta-analysis to derive the potential relationship of the target SNP with cancer susceptibility after integrating data from all eligible studies. This meta-analysis of nine studies containing 5078 patients with PC and 5952 controls systematically evaluated the association between a functional polymorphism within the COX-2 promoter, -765G>C (rs20417), and $\mathrm{PC}$ risk. Overall results from all genetic models tested consistently suggested that COX2 $-765 \mathrm{G}>\mathrm{C}$ polymorphism did not constitute a conspicuous risk factor in the susceptibility of PC. Subgroup analysis by ethnicity also demonstrated similar results. These internally consistent results suggested that $C O X 2-765 \mathrm{G}>\mathrm{C}$ might have no impact on the pathogenesis of PC. Previous meta-analyses have shown an association between the COX2 -765G >C polymorphism and cancer risk in gastric (Zhao et al., 2014), esophageal (Liang et al., 2011), and colorectal cancers (Wang et al., 2013). One possible explanation for this discrepancy is that different types of cancer have various mechanisms of carcinogenesis. Although preclinical research has suggested that the COX2 -765G>C polymorphism might be related to PC risk (Wu et al., 2011; Catsburg et al., 2012; Joshi et al., 2012), the results from our study indicated that COX2 -765G>C was unlikely to be associated with PC risk. Since Panguluri et al. (2004) first claimed that this SNP played a role in the development of PC, considerable research has been conducted in this field. However, most of the studies failed to obtain a significant result. Furthermore, a stratified analysis by ethnicity was conducted herein, and a null significant association was found, with the exception of the homozygote comparison (CC vs GG) and dominant model (GC+CC vs GG) analyses of Asian populations, in which $C O X 2-765 G>C$ was positively associated with $\mathrm{PC}$ risk. However, as this result is, to our knowledge, unique among published studies, we still need further preclinical research to confirm the possibility that the COX2 -765G>C variant may have an association with $\mathrm{PC}$ risk in Asian populations.

The meta-analysis presented here demonstrated several strengths. For COX2 -765G>C and PC risk, no significant heterogeneity was observed in all the genetic models. In addition, we did not identify any publication bias for our meta-analysis. Furthermore, our sample size was sufficient to provide adequate statistical power for our analyses, which strengthened the reliability of our results. However, potential limitations of this study should also be addressed. First, our results were based on unadjusted estimates, and a more precise evaluation stratified by age, gender, cigarette consumption, family history, and environmental exposures could be performed if individual data are available. Second, only published full-text articles were included in the current meta-analysis, which might cause publication bias even though both Begg's and Egger's tests did not confirm the existence of bias. Finally, the obtained results might potentially be false negatives, as some of the individual studies included in the current meta-analysis reported a significant association of the COX2 -765G >C polymorphism with $P C$ risk.

Despite these limitations, this meta-analysis has provided reliable results based on ample study power. In summary, our study demonstrated that COX2 -765G>C (rs20417) is unlikely to be associated with PC risk. However, additional well-designed and unbiased studies, with larger sample size, in diverse ethnic populations, and with the consideration of gene-gene and geneenvironment interactions are still required to resolve this issue in the future. 


\section{ACKNOWLEDGMENTS}

Yan-Qing Feng and Yu Li contributed equally to this study for designing and performing the research. Yan-Qing Feng and Yu Li, analyzed the data. Yan-Qing Feng wrote the paper. Wei-Dong Xiao, Yong Li and Gong-Xian Wang reviewed and revised the paper. Research supported by the National Natural Science Foundation of China (Grant \#91229119), the Natural Science Foundation of Jiangxi (Grant \#800GZY0039), and the Science and Technology Development Fund of Macao Special Administrative Region (Grant \#064/2012/A).

\section{REFERENCES}

Agachan Cakmakoglu B, Attar R, Kahraman OT, Dalan AB, et al. (2011). Cyclooxygenase-2 gene and epithelial ovarian carcinoma risk. Mol. Biol. Rep. 38: 3481-3486.

Balistreri CR, Caruso C, Carruba G, Miceli V, et al. (2010). A pilot study on prostate cancer risk and pro-inflammatory genotypes: pathophysiology and therapeutic implications. Curr. Pharm. Des. 16: 718-724.

Begg CB and Mazumdar M (1994). Operating characteristics of a rank correlation test for publication bias. Biometrics 50: 1088-1101.

Bye H, Prescott NJ, Matejcic M, Rose E, et al. (2011). Population-specific genetic associations with oesophageal squamous cell carcinoma in South Africa. Carcinogenesis 32: 1855-1861.

Catsburg C, Joshi AD, Corral R, Lewinger JP, et al. (2012). Polymorphisms in carcinogen metabolism enzymes, fish intake, and risk of prostate cancer. Carcinogenesis 33: 1352-1359.

Chen WS, Wei SJ, Liu JM, Hsiao M, et al. (2001). Tumor invasiveness and liver metastasis of colon cancer cells correlated with cyclooxygenase-2 (COX-2) expression and inhibited by a COX-2-selective inhibitor, etodolac. Int. J. Cancer 91: 894-899.

Cheng I, Liu X, Plummer SJ, Krumroy LM, et al. (2007). COX2 genetic variation, NSAIDs, and advanced prostate cancer risk. Br. J. Cancer 97: 557-561.

Cochran WG (1954). The combination of estimates from different experiments. Biometrics 10: 101-129.

Cocoş R, Schipor S, Nicolae I, Thomescu C, et al. (2012). Role of COX-2 activity and CRP levels in patients with nonmelanoma skin cancer. -765G>C PTGS2 polymorphism and NMSC risk. Arch. Dermatol. Res. 304: 335-342.

Coskunpinar E, Eraltan IY, Turna A and Agachan B (2011). Cyclooxygenase-2 gene and lung carcinoma risk. Med. Oncol. 28: 1436-1440.

Daraei A, Salehi R and Mohamadhashem F (2012). PTGS2 (COX2) -765G>C gene polymorphism and risk of sporadic colorectal cancer in Iranian population. Mol. Biol. Rep. 39: 5219-5224.

DerSimonian R and Laird N (1986). Meta-analysis in clinical trials. Control Clin. Trials 7: 177-188.

Ding XZ, Tong WG and Adrian TE (2000). Blockade of cyclooxygenase-2 inhibits proliferation and induces apoptosis in human pancreatic cancer cells. Anticancer Res. 20: 2625-2631.

Dossus L, Kaaks R, Canzian F, Albanes D, et al. (2010). PTGS2 and IL6 genetic variation and risk of breast and prostate cancer: results from the Breast and Prostate Cancer Cohort Consortium (BPC3). Carcinogenesis 31: 455-461.

Duval S and Tweedie R (2000). Trim and fill: a simple funnel-plot-based method of testing and adjusting for publication bias in meta-analysis. Biometrics 56: 455-463.

Egger M, Davey Smith G, Schneider M and Minder C (1997). Bias in meta-analysis detected by a simple, graphical test. BMJ 315: 629-634.

He J, Zhang Q, Ren Z, Li Y, et al. (2012). Cyclooxygenase-2-765 G/C polymorphisms and susceptibility to hepatitis B-related liver cancer in Han Chinese population. Mol. Biol. Rep. 39: 4163-4168.

Joshi AD, Corral R, Catsburg C, Lewinger JP, et al. (2012). Red meat and poultry, cooking practices, genetic susceptibility and risk of prostate cancer: results from a multiethnic case-control study. Carcinogenesis 33: 2108-2118.

Kawata R, Reddy ST, Wolner B and Herschman HR (1995). Prostaglandin synthase 1 and prostaglandin synthase 2 both participate in activation-induced prostaglandin D2 production in mast cells. J. Immunol. 155: 818-825.

Li M, Wu X and Xu XC (2001). Induction of apoptosis in colon cancer cells by cyclooxygenase-2 inhibitor NS398 through a cytochrome c-dependent pathway. Clin. Cancer Res. 7: 1010-1016.

Li Y, Dai L, Zhang J, Wang P, et al. (2012). Cyclooxygenase-2 polymorphisms and the risk of gastric cancer in various degrees of relationship in the Chinese Han population. Oncol. Lett. 3: 107-112.

Liang Y, Liu JL, Wu Y, Zhang ZY, et al. (2011). Cyclooxygenase-2 polymorphisms and susceptibility to esophageal cancer: a meta-analysis. Tohoku J. Exp. Med. 223: 137-144. 
Mantel $\mathrm{N}$ and Haenszel W (1959). Statistical aspects of the analysis of data from retrospective studies of disease. J. Nat/. Cancer Inst. 22: 719-748.

Masferrer JL, Leahy KM, Koki AT, Zweifel BS, et al. (2000). Antiangiogenic and antitumor activities of cyclooxygenase-2 inhibitors. Cancer Res. 60: 1306-1311.

Monroy CM, Cortes AC, Lopez MS, D'Amelio AM Jr, et al. (2011). Hodgkin disease risk: role of genetic polymorphisms and gene-gene interactions in inflammation pathway genes. Mol. Carcinog. 50: 36-46.

Murad A, Lewis SJ, Smith GD, Collin SM, et al. (2009). PTGS2-899G.C and prostate cancer risk: a population-based nested case-control study (ProtecT) and a systematic review with meta-analysis. Prostate Cancer Prostatic Dis. 12: 296-300.

Pandey S, Mittal RD, Srivastava M, Srivastava K, et al. (2010). Cyclooxygenase-2 gene polymorphisms and risk of cervical cancer in a North Indian population. Int. J. Gynecol. Cancer 20: 625-630.

Panguluri RC, Long LO, Chen W, Wang S, et al. (2004). COX-2 gene promoter haplotypes and prostate cancer risk. Carcinogenesis 25: 961-966.

Papafili A, Hill MR, Brull DJ, McAnulty RJ, et al. (2002). Common promoter variant in cyclooxygenase-2 represses gene expression: evidence of role in acute-phase inflammatory response. Arterioscler. Thromb .Vasc. Biol. 22: 1631-1636.

Peters WH, Lacko M, Te Morsche RH, Voogd AC, et al. (2009). COX-2 polymorphisms and the risk for head and neck cancer in white patients. Head Neck 31: 938-943.

Piranda DN, Festa-Vasconcellos JS, Amaral LM, Bergmann A, et al. (2010). Polymorphisms in regulatory regions of cyclooxygenase-2 gene and breast cancer risk in Brazilians: a case-control study. BMC Cancer 10: 613.

Sheng H, Shao J, Washington MK, DuBois RN (2001). Prostaglandin E2 increases growth and motility of colorectal carcinoma cells. J. Biol. Chem. 276: 18075-18081.

Siegel R, Naishadham D and Jemal A (2012). Cancer statistics, 2012. CA Cancer J. Clin. 62: 10-29.

Szczeklik W, Sanak M and Szczeklik A (2004). Functional effects and gender association of COX-2 gene polymorphism G-765C in bronchial asthma. J. Allergy Clin. Immunol. 114: 248-253.

Wang J, Guo X, Zhang J, Song J, et al. (2013). Cyclooxygenase-2 polymorphisms and susceptibility to colorectal cancer: a meta-analysis. Yonsei Med. J. 54: 1353-1361.

Wang MT, Honn KV and Nie D (2007). Cyclooxygenases, prostanoids, and tumor progression. Cancer Metastasis Rev. 26: 525-534.

Wu HC, Chang CH, Ke HL, Chang WS, et al. (2011). Association of cyclooxygenase 2 polymorphic genotypes with prostate cancer in Taiwan. Anticancer Res. 31: 221-225.

Zhao D, Xu D, Zhang X, Wang L, et al. (2009). Interaction of cyclooxygenase-2 variants and smoking in pancreatic cancer: a possible role of nucleophosmin. Gastroenterology 136: 1659-1668.

Zhao F, Zhu H, Huang M, Yi C, et al. (2014). The 765G>C polymorphism in the cyclooxygenase-2 gene and gastric cancer risk: an update by meta-analysis. Asian Pac. J. Cancer Prev. 15: 2863-2868.

Zheng J, Chen S, Jiang L, You Y, et al. (2011). Functional genetic variations of cyclooxygenase-2 and susceptibility to acute myeloid leukemia in a Chinese population. Eur. J. Haematol. 87: 486-493. 